\title{
Topical cyclosporin A in the management of vernal keratoconjunctivitis
}

\author{
Abstract \\ Purpose To study the efficacy of topical \\ cyclosporin $A$ in children with vernal \\ keratoconjunctivitis in a prospective \\ randomised double-masked trial. \\ Methods Twenty-four children between 5 and \\ 16 years of age were randomly treated with \\ cyclosporin A (CsA) and placebo (vehicle) and \\ followed up for 4 months. \\ Results Of the 12 patients given CsA, 11 \\ showed symptomatic improvement by 7 days \\ of starting treatment whereas only 3 patients \\ in the placebo group showed mild \\ symptomatic improvement. \\ Conclusions Topical cyclosporin is a safe and \\ effective treatment in vernal \\ keratoconjunctivitis.
}

Key words India, Placebo-controlled trial, Topical cyclosporin, Vernal keratoconjunctivitis

Vernal keratoconjunctivitis is a seasonal form of allergic conjunctivitis associated with severe itching, photophobia, redness and tenacious discharge affecting children and young adults. Remission generally occurs in the late teens. However, the symptoms can hamper the normal activities of a child and are of great concern to the parents. Although the condition may be seasonal, chronic cases may have persistent symptoms throughout the year, with some developing corneal complications.

Prolonged use of topical steroids for treatment also leads to corneal ulceration and glaucoma in a number of children.

Histopathological studies have proven the presence of a CD4 ${ }^{+}$subset of T lymphocytes in the conjunctival papillae in patients with vernal keratoconjunctivitis. This subset of lymphocytes is known to produce interleukin-2. Cyclosporin is an immunomodulator which not only inhibits the release of interleukins but has also been found to inhibit eosinophil activation and the release of granule proteins. Hence we carried out a randomised placebo-controlled trial to assess the efficacy of topical cyclosporin A (CsA) in vernal keratoconjunctivitis.

\section{Subjects and methods}

Twenty-four children ( 22 boys, 2 girls) between the ages of 5 and 16 years with classic symptoms of active vernal keratoconjunctivitis were enrolled in the study between May and September 1999. A detailed history of symptoms and other allergies was recorded. Slit-lamp examination was performed to assess the severity of conjunctival and corneal involvement. After discontinuing their previous medication the children were re-examined after 5 days. Only patients with a minimum of 2 months follow-up were included in the study. They were assigned (by computer-generated randomisation) to receive either CsA $2 \%$ in olive oil or placebo (plain olive oil). Both the drops were to be used four times daily in both eyes. Only the pharmacist, who was kept independent of the trial, knew the coding of the vials. The drops were dispensed in identical bottles with their code written on the bottle. Both the doctor and the patients were masked to the identity of the contents. Informed consent was obtained from the parents after explaining the relative safety of the medication and the possible side-effects.

Patients were followed up every week for the first month and every month thereafter for 3 months. The severity of itching, redness, tearing and photophobia along with mucus discharge were to be noted by the parents along with any medication-related side-effects. The subjective and objective assessment scale used by us was a 5-point scale based on the study of Ben Ezra et al. ${ }^{1}$ (Table 1). Patients were designated treatment failures if they did not show any change in their symptoms (grade 2) or had a worsening of their symptoms (grade 1) even after 2 months of therapy.

\section{Statistical analysis}

We used Fisher's (two-tailed) exact test for the dichotomous data for assessment of the ratings by the patients. Continuous variables were compared by a two-sample $t$-test.

\author{
V. Gupta \\ P.K. Sahu \\ Dr R.P. Centre for \\ Ophthalmic Sciences \\ All India Institute of Medical \\ Sciences \\ New Delhi 110029, India \\ Dr Viney Gupta \\ Dev Eye Hospital \\ Jagadhri Road \\ Ambala Cantt 133001 \\ Harayana, India \\ Tel: +91 171652822 \\ e-mail: gupta_v@mailcity.com
}

Received: 31 May 2000

Accepted in revised form:

5 September 2000 


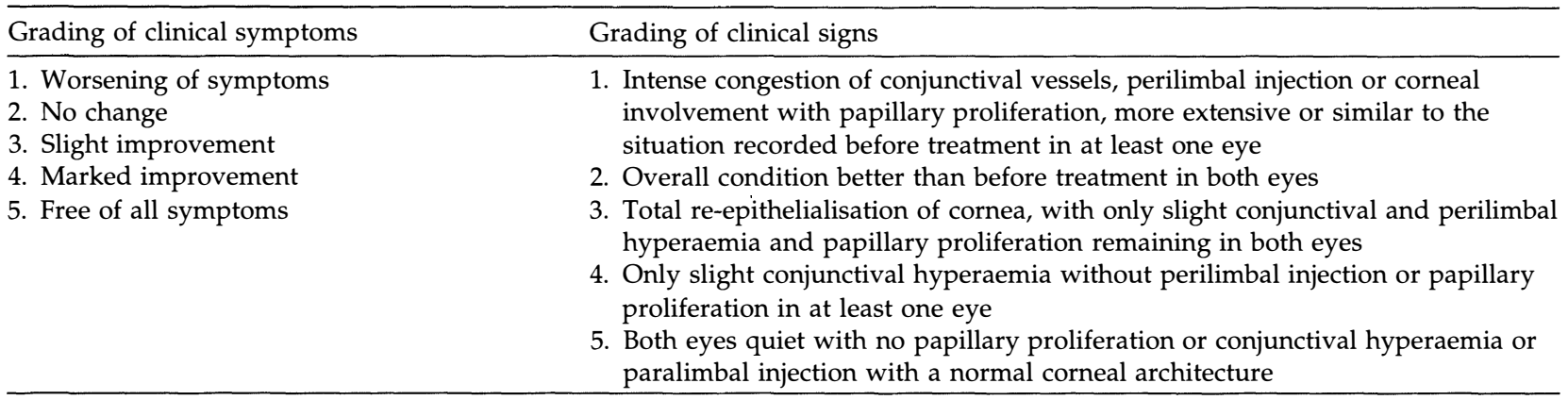

\section{Results}

There were 10 boys and 2 girls in the CsA group and 12 boys in the placebo group. The average age of the patients in the CsA group was $10.8 \pm 3.5$ years and the average age in the placebo group was $10.6 \pm 4.6$ years. On average the symptoms of all patients had been present for $10 \pm 4$ days. None of the patients had any other associated allergic condition and, except for 2 patients with a family history of asthma, no child had any history of allergy in the family. Two patients in the CsA group and 3 in the placebo group had limbal verbal keratoconjunctivitis, the rest had tarsal conjunctivitis. Before starting treatment, 5 patients in the CsA group and 4 patients in placebo group were on topical steroids which were stopped 5 days before starting topical CsA. Two boys in the CsA group had corneal involvement with shield ulcer which took 2 months to heal. These 2 patients were given topical antibiotics in addition to CsA therapy. Though they both noticed marked improvement of their symptoms, they continued to show signs of chronic vernal keratoconjunctivitis.

Eleven of the $12(91 \%)$ patients in the CsA group had mild to marked improvement in symptoms which became evident by the first week of follow-up, in contrast to only 4 of the $12(25 \%)$ in the placebo group who showed mild improvement of their symptoms $(p<0.05)$ (Fig. 1). One patient in the CsA group who did not show any change in his symptoms even after 2 months was put on $4 \%$ sodium cromoglycate and $0.1 \%$ dexamethasone eye drops. The 9 patients in the placebo group who were deemed treatment failures were also offered alternative treatment.

Eight of 12 patients $(66 \%)$ in the CsA group showed varying degrees of papillary regression and 4 of these patients were disease-free at the 4 month follow-up. However, only 2 patients $(16 \%)$ in the placebo group had mild improvement in their clinical signs $(p<0.05)$ (Fig. 2).
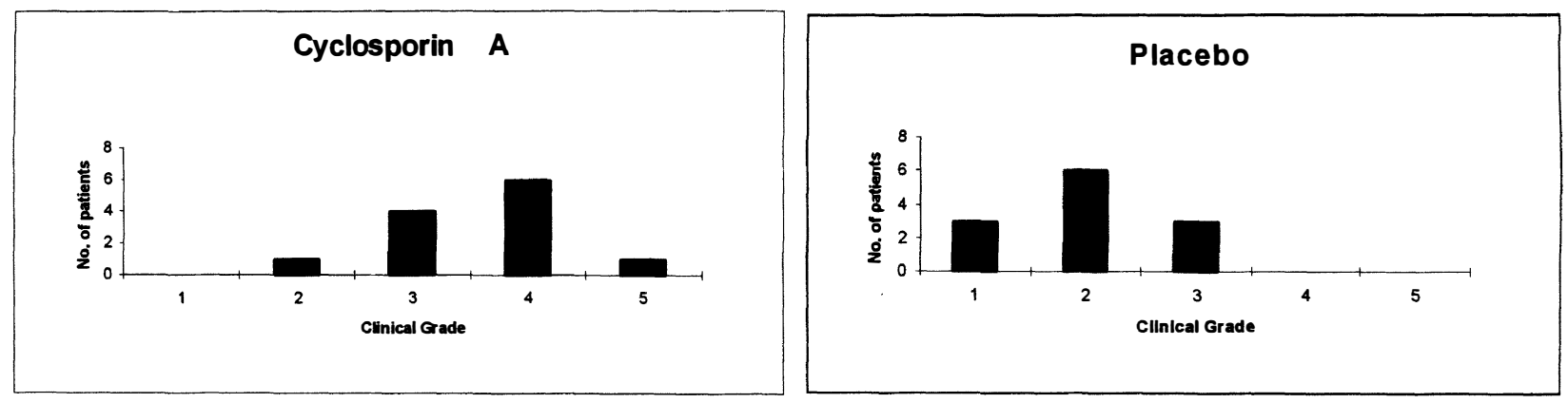

Fig. 1. Overall rating of clinical symptoms at 4 month follow-up.
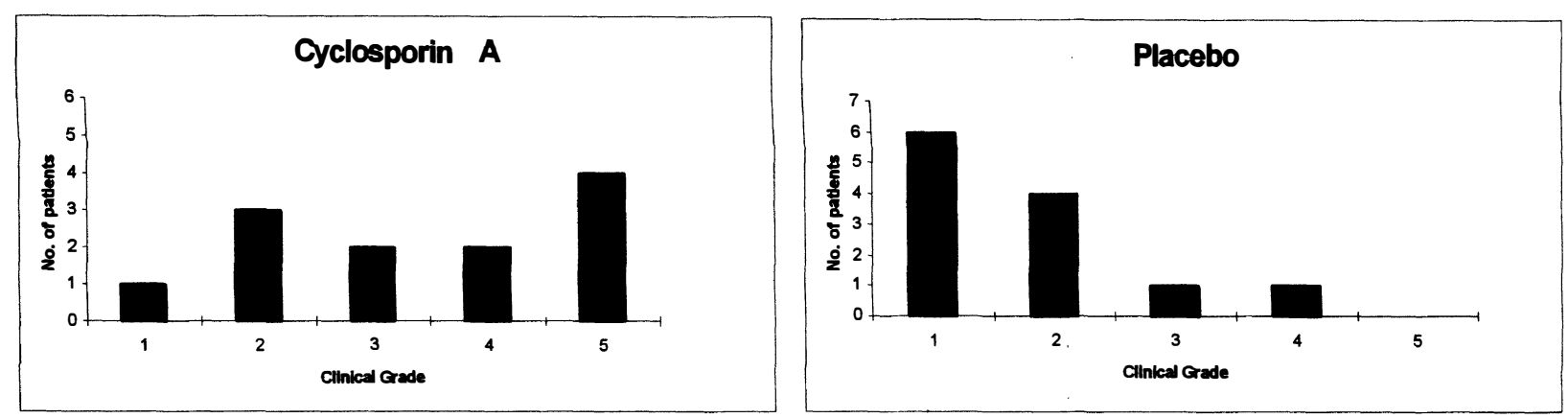

Fig. 2. Overall rating of clinical signs at 4 month follow-up. 


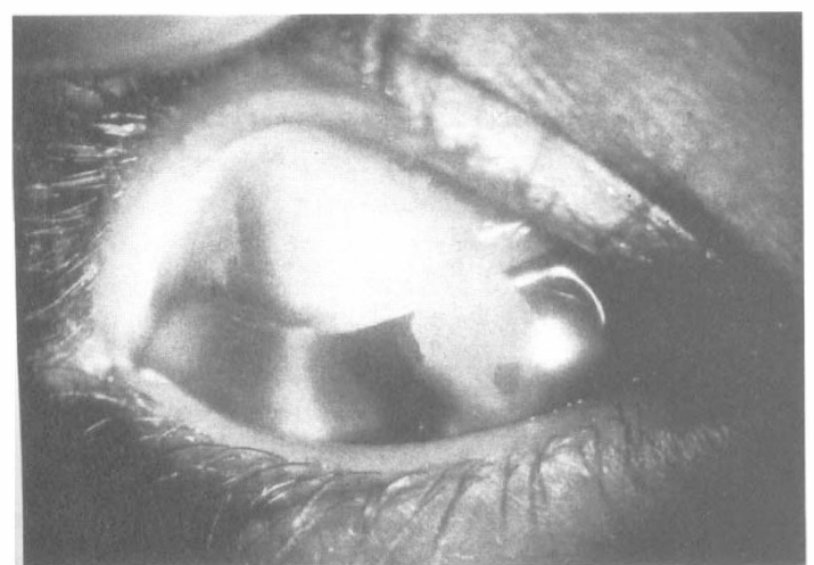

Fig. 3. Healed papillary proliferations after 3 months of cyclosporin therapy in a 6-year-old child with shield ulcer.

The most common side-effect was stinging (10 CsA, 11 placebo) and redness of the eyes ( $6 \mathrm{CsA}, 11$ placebo) a few minutes after instilling the medication. Other sideeffects included tearing ( $6 \mathrm{CsA}, 8$ placebo) and transient blurring of vision (1 CsA, 3 placebo). None of the patients developed an allergic reaction or CsA-related corneal epitheliopathy. Side-effects were not severe enough for any patient to discontinue the medication.

The 2 boys in the CsA group with shield ulcer (Fig. 3) received treatment for 5 months without any complaints severe enough to require discontinuation of the drops.

\section{Discussion}

Apart from topical steroids and $2 \%$ sodium cromoglycate, several other therapeutic agents including topical mast cell stabiliser, lodoxamide, ${ }^{2}$ topical NSAID ${ }^{3}$ and even aspirin ${ }^{4}$ have been used with varying success. In our study $91 \%$ patients showed decreased symptoms and $66 \%$ showed definitive improvement of their clinical signs, with the majority noticing a benefit by the first week of therapy with topical CsA. As noted in Figs. 1 and 2 patients felt symptomatically better yet their signs did not show the same degree of improvement. This has also been noted in the short-term study of 11 patients by Secchi et al. ${ }^{5}$

Since more patients in the placebo group experienced side-effects after instillation of drops we attribute them to the olive oil used as a vehicle to dissolve the cyclosporin powder. The beneficial effects noticed by some patients in the placebo group could also be attributed to the lubricating effect of olive oil. Agents that have been used by other authors are castor oil, ${ }^{5}$ maize oil $^{6}$ and corn oil. ${ }^{7}$

Topical cyclosporin has been used previously for vernal keratoconjunctivitis in a pilot study by Ben Ezra et al. ${ }^{1}$ Eleven of their 12 patients showed improvement in the first week of starting treatment. However, results at 2 month follow-up were not encouraging as only 3 patients remained free of disease. This was probably due to the fact that they recruited patients with severe vernal keratoconjunctivitis who had been symptomatic for from 2 to 9 years. Our patients were fresh cases of vernal keratoconjunctivitis who showed definitive improvement with CsA over a 4 month follow-up period.

In a tropical country such as India with a very high incidence of vernal keratoconjunctivitis, especially in the summer months, and with many eyes being lost due to steroid-induced glaucoma or corneal ulceration secondary to steroids, cyclosporin seems to be an effective modality of treatment with few side-effects. A long-term study of the efficacy of cyclosporin in chronic vernal keratoconjunctivitis is thus being planned.

\section{References}

1. BenEzra D, Peer J, Brodsky M, Cohen E. Cyclosporine eyedrops for the treatment of severe vernal keratoconjunctivitis. Am J Ophthalmol 1986;101:278-82.

2. Caldwell DR, Verin P. Efficacy and safety of lodoxamide $0.1 \%$ versus cromolyn sodium $4 \%$ in patients with vernal keratoconjunctivitis. Eye 1992;113:632-7.

3. Buckley DC, Caldwell DR, Reaves TA. Treatment of vernal keratoconjunctivitis with suprofan, a topical non-steroidal anti-inflammatory agent. Invest Ophthalmol Vis Sci 1993;38:133-40.

4. Abelson MB, Butrus SI, Weston JH. Aspirin therapy in vernal conjunctivitis. Am J Ophthalmol 1983;95:502.

5. Secchi A, Tognon S, Leonardi A. Topical use of cyclosporine in the treatment of vernal keratoconjunctivitis. Am J Ophthalmol 1990;110:641-5.

6. Hingorani M, Moodaley L, Calder VL, Buckley RJ, Lightman S. A randomised, placebo-controlled trial of topical cyclosporin A in steroid dependent atopic keratoconjunctivitis. Ophthalmology 1998;105:1715-20.

7. Bleik JH, Tabbara KF. Topical cyclosporine in vernal keratoconjunctivitis. Ophthalmology 1991;98:1679-84. 